\title{
Monte Carlo Simulations for the Magnetic Phase Diagram of the Double Exchange Hamiltonian
}

\author{
M.J. Calderón and L. Brey \\ Instituto de Ciencia de Materiales (CSIC). Campus de la Universidad Autónoma, 28049, Madrid, Spain.
}

(May 9, 2018)

We have used Monte Carlo simulation techniques to obtain the magnetic phase diagram of the double exchange Hamiltonian. We have found that the Berry phase of the hopping amplitude has a negligible effect in the value of the magnetic critical temperature. To avoid finite size problems in our simulations we have also developed an approximated expression for the double exchange energy. This allows us to obtain the critical temperature for the ferromagnetic to paramagnetic transition more accurately. In our calculations we do not observe any strange behavior in the kinetic energy, chemical potential or electron density of states near the magnetic critical temperature. Therefore, we conclude that other effects, not included in the double exchange Hamiltonian, are needed in order to understand the metal-insulator transition which occurs in the manganites.

PACS number 71.10.-w, 75.10.-b

\section{INTRODUCTION}

The recent discovery of colossal magnetoresistance (CMR) [1] in mixed-valence compounds of the form $L a_{1-x}^{3+} A_{x}^{2+} M n_{1-x}^{3+} M n_{x}^{4+} O_{3}^{2-}$, (where $A$ can be Ca, Sr or $\mathrm{Ba})$ has revived the interest in these materials with perovskite structure. The interest is focused on the phase diagram and the magneto-transport properties. Both features have been determined experimentally [2]. For $0.1 \leq x \leq 0.5$ and low temperatures the system is metallic and presents ferromagnetic order. As the temperature increases the system becomes insulator and paramagnetic. The ferro-paramagnetic transition occurs at an $x$ dependent critical temperature $T_{c}(x) \sim 300 K$. The colossal magnetoresistance effects are observed for temperatures close to $T_{c}(x)$. For $x \leq 0.1$ and low temperatures the system is a 'layer' antiferromagnetic with ferromagnetic coupling inside planes.

In these compounds the electronically active orbitals are the $M n d$ orbitals. The number of $d$ electrons per $M n$ is $x+3$, namely, four electrons per $M n^{3+}$ and three per $M n^{4+}$. The cubic symmetry and the strong Hund's rule coupling make that three electrons get trapped in the $t_{2 g}$ states and therefore these electrons become electrically inert, forming a core spin $S$ of magnitude $3 / 2$. The rest of the electrons go to the $e_{g}$ orbitals and they get strongly coupled to $S$ by the Hund's rule coupling. Consequently, all the spins on each $M n$ prefer to be parallel. For small values of $x$, the perovskites show a long range Jahn-Teller order which selects a preferred $d$ orbital and therefore it is possible to assume that the electrons move only through one $d$ orbital.

The double exchange (DE) mechanism developed by Zener [3 5], explains the existence of ferromagnetism and metallic behavior at low temperatures. In this model, the electrons get mobility between the manganese ions using oxygen, which is magnetically inert, as an intermediate. As a consequence, the tunneling takes place between two configurations in which the $M n$ ions of different charge $\left(M n^{3+}\right.$ and $\left.M n^{4+}\right)$ interchange their valence states. This conduction process is proportional to the electron transfer integral $t$ if the core spins of $M n^{3+}$ and $M n^{4+}$ are aligned. Otherwise, the transfer integral is inversely proportional to the Hund's rule coupling energy, which for the $M n$ ions is much larger than $t$. In the DE model the ferromagnetism is then induced via this electron conduction process.

In the limit of large Hund's coupling the spin of the electron in the active $d$ orbital is parallel to the local core spin $\mathbf{S}_{i}$, which is treated as a classical rotator with the normalization $|\mathbf{S}|=1$ and characterized by the angles $\theta_{i}$ and $\phi_{i}$. Then, semiclassically, the effective hopping Hamiltonian is

$$
H=-\sum_{\langle i, j\rangle}\left(t_{i, j} C_{i}^{+} C_{j}+\text { h.c. }\right)
$$

Here $C_{i}^{+}$creates an electron at site $i$ with spin parallel to $\mathbf{S}_{i},\langle i, j\rangle$ denotes the nearest-neighbor pairs, which are only counted once, and the hopping amplitude acquires a Berry's phase and it becomes a complex number given by [6],

$$
t_{i, j}=t\left(\cos \frac{\theta_{i}}{2} \cos \frac{\theta_{j}}{2}-\sin \frac{\theta_{i}}{2} \sin \frac{\theta_{j}}{2} e^{i\left(\phi_{i}-\phi_{j}\right)}\right)
$$

This complex hopping appears after rotating the conduction electron spins so that the spin quantization axis at site $i$ is parallel to $\mathbf{S}_{i}$, and then project onto the spin parallel to $\mathbf{S}_{j}$. In this paper we will call complex DE (cDE) model the system governed by the Hamiltonian (1) with the hopping given by (2).

For topologies where electrons close paths do not occur, it is possible to choose wave functions phase factors such that

$$
t_{i, j} \rightarrow\left|t_{i, j}\right|=t \cos \frac{\theta_{i, j}}{2}
$$

being $\theta_{i, j}$ the angle between the semiclassical spins $\mathbf{S}_{i}$ and $\mathbf{S}_{j}$. 
In this work we will use the name DE model for the system controlled by equation (11) with the hopping (3). This is the Hamiltonian proposed by Anderson and Hasegawa [4] as a generalization of the Hamiltonian describing the tunneling between two fixed sites $i$ and $j$.

De Gennes [5] and Kubo and Ohata [7] calculated, in a mean field theory approximation, the magnetic phase diagram of the DE model. Their results show a ferroparamagnetic phase transition at a critical temperature

$$
\frac{T_{c}^{M F}}{t}=1.6\left\langle C_{i}^{+} C_{j}\right\rangle_{0}
$$

This transition is accompanied by a change in the temperature dependence of the resistivity. In equation (4), $\langle\hat{O}\rangle_{0}$ means the expectation value of the operator $\hat{O}$ at zero temperature.

However, recently it has been pointed out 8 that the critical temperatures predicted by the mean field theory of the DE model is much bigger than the observed experimentally. Furthermore, the resistivity implied by the hopping (3) is incompatible with many aspects of the experimental information. Millis et al [8] proposed that in addition to the DE mechanism a strong electron-phonon interaction plays a crucial role in these perovskites. The interplay between these two effects could reproduce the experimental critical temperature and the observed behavior of the resistivity at the magnetic transition temperature [9,10].

On the other hand, Yunoki et al 11] claim that the inclusion of the phase in the hopping term can lower the critical temperature down to the experimental values. Also, for large but finite Hund's coupling, they obtain that at low concentrations phase separation between hole-poor antiferromagnetic and hole-rich ferromagnetic regions occurs. The existence of phase separation is also obtained by solving the DE model plus an antiferromagnetic coupling between next neighbors core spins [12].

In this paper we are interested in two points; first, in comparing the magnetic phase diagram of the two DE models, equations (2) and (3), and second, in obtaining an approximated expression for the DE energy which allows us to perform Monte Carlo calculations in bigger unit cells and to obtain accurate values of the magnetic critical temperature. The main results of our calculations are shown in Fig.11. Here we show the critical temperature versus concentration, for the complex DE model and for the real DE model, in the case of a unit cell of size $4 \times 4 \times 4$. We obtain that there is not big difference between the critical temperatures of both models. This result indicates that the contribution to the partition function of electronic configurations in which fermions move on closed loops in real space can be neglected. In Fig.1 we also show Monte Carlo results obtained by using an approximated expression for the electron kinetic energy. We have checked in small systems that this approximation is realistic. From our results we obtain that the critical temperature of the DE model is in the range of the experimental one [2].
The paper is organized as follows: in section II we describe briefly the Monte Carlo algorithm we use and in section III we present the results obtained for the cDE and DE Hamiltonians. In section IV we introduce an approximation to the DE energy which allows us to perform Monte Carlo simulations in bigger systems. Section $\mathrm{V}$ is devoted to show the results obtained in the frame of this approximation. Finally, we present the conclusions in section VI.

\section{DESCRIPTION OF THE MONTE CARLO ALGORITHM}

For calculating the magnetic phase diagrams, we have performed classical Monte Carlo (MC) simulations on the classical core spin angles. The simulations are done in $N \times N \times N$ cubic lattices with periodic boundary conditions. Although the localized spins are considered classical, the kinetic energy of the conduction electrons is calculated by diagonalizing the DE Hamiltonian because we consider it as a quantum quantity.

The standard Metropolis algorithm 13. was used in the MC simulations. The sites to be considered for a change in the spin orientation are randomly chosen. Once a site is selected for a spin reorientation, the angle associated with an attempted change of the spin is chosen at random from within a specified range [14]. Then the energy change, $\Delta E$, associated with the attempted update, is calculated. If the quantity $\exp (-\Delta E / T)$ is smaller than a random number between 0 and 1, the change is allowed, otherwise, it is rejected. Typically, 5000-7000 MC steps per spin are used for equilibration and 3000-5000 steps for spin are used for calculating averages.

In the simulations we calculate the average of the internal energy, $E$, and the average of the absolute value of the magnetization, $M$,

$$
\begin{gathered}
E=\frac{1}{N^{3}}\langle H\rangle, \\
M=\frac{1}{N^{3}}\left\langle\left|\sum_{i} \mathbf{S}_{\mathbf{i}}\right|\right\rangle,
\end{gathered}
$$

where \langle\rangle denotes statistical average. Since the MC updating procedure generates uniform rotations of the spin system, a calculation of the MC average of the direction of the magnetization is not meaningful. Also we calculate the average value of the width of the density of states, $W$, and the value of the chemical potential, $\mu$, measured with respect to the bottom of the density of states,

$$
\begin{aligned}
W & =\left\langle\varepsilon_{\max }-\varepsilon_{\min }\right\rangle \\
\mu & =\left\langle\varepsilon_{o c c}-\varepsilon_{\min }\right\rangle,
\end{aligned}
$$

where $\varepsilon_{\min }$ and $\varepsilon_{\max }$ are the minimum and the maximum energies obtained from diagonalizing the electron Hamiltonian, and $\varepsilon_{o c c}$ is the higher energy of the occupied states. 


\section{MONTE CARLO RESULTS FOR THE DOUBLE EXCHANGE MODELS}

In order to obtain the internal energy in each of the Monte Carlo steps, it is necessary to diagonalize the electronic Hamiltonian. The diagonalization is very expensive in terms of CPU time, and therefore we can only study small size systems. Here we present the results obtained for a cubic unit cell of size $N=4$ and for the electron concentrations $x=0.1,0.25$ and 0.3 .

In figure 2, it is presented, for the $\mathrm{DE}$ and the $\mathrm{cDE}$ Hamiltonians, the magnetization, $M$, as a function of the temperature, $T$. Due to the finite size of the unit cells used in the simulations, $M$ is different from zero at any temperature, and we define the critical temperature, $T_{c}$, as the point where the second derivative of $M$ with respect $T$ changes sign. This way of obtaining the critical temperatures, implies uncertainties of around $10 \%$ of the value of $T_{c}$. In any case we obtain that the critical temperatures of the DE and the cDE Hamiltonians are practically the same. In figure 3 we plot the internal energy $E$ as a function of $T$. Note that the difference between the internal energies obtained by using the cDE and using the DE Hamiltonians is rather small. That is the reason why both models give very similar magnetic critical temperatures.

These results imply that in order to calculate total kinetic energies the close paths are almost irrelevant.

In figure 4 we plot, as a function of the temperature, the width of the density of states $W$ and the chemical potential $\mu$, for the DE case. Note that at temperatures close to $T_{c}$, the bandwidth and the chemical potential have a value bigger than the obtained in the fully disordered case, $T \rightarrow \infty$. This is because in the DE models the ferromagnetic to paramagnetic transition is a second order phase transition, and therefore the internal energy is continuous at the critical temperature. Moreover, the bandwidth and the chemical potential present a continuous behavior near $T_{c}$. In the DE models we do not observe any change in the electronic states at the ferroparamagnetic critical temperature.

\section{APPROXIMATION FOR THE DOUBLE EXCHANGE ENERGY}

In the MC calculations of the DE models, the size of the matrix to diagonalize impose a restriction on the dimension of the unit cell used in the simulation. In order to be able to perform simulations in bigger systems, we have developed a second order perturbation theory for obtaining an expression for the electron kinetic energy in a background of randomly oriented core spins.

We start writing the Hamiltonian (11) in the form,

$$
H \equiv H_{0}+V
$$

$$
\begin{aligned}
H_{0} & \equiv-\bar{t} \sum_{\langle i, j\rangle}\left(C_{i}^{+} C_{j}+\text { h.c. }\right) \\
V & \equiv-\sum_{\langle i, j\rangle}\left(\delta t_{i, j} C_{i}^{+} C_{j}+\text { h.c. }\right),
\end{aligned}
$$

being $\bar{t}$ the average of the absolute value of the hopping amplitude,

$$
\bar{t}=\frac{1}{3 N_{0}} \sum_{\langle i, j\rangle}\left|t_{i, j}\right|,
$$

and

$$
\delta t_{i, j}=t_{i, j}-\bar{t}
$$

Here $N_{0}$ is the total number of $M n$ ions in the system. Note that since the Hamiltonian is hermitic, $\bar{t}$ is a real quantity.

Given a disordered system, characterized by a set of $\left\{t_{i, j}\right\}$, we want to obtain the expectation value of $H$. The Hamiltonian $H_{0}$ can be diagonalized by using the Bloch states,

$$
|\mathbf{k}\rangle=\frac{1}{\sqrt{N_{0}}} \sum_{i} e^{i \mathbf{k} \cdot \mathbf{R}_{\mathbf{i}}}|i\rangle,
$$

where $|i\rangle$ represents the atomic orbital at site $i, \mathbf{R}_{\mathbf{i}}$ are the lattice vectors and $\mathbf{k}$ is a wave vector in the first Brillouin zone. The energy of the state $|\mathbf{k}\rangle$ is

$$
\varepsilon(\mathbf{k})=-2 \bar{t}\left(\cos k_{x} a+\cos k_{y} a+\cos k_{z} a\right),
$$

being $a$ the lattice parameter. In function of these eigenvalues, the expectation value of $H_{0}$ is,

$$
E_{0}=\sum_{k}^{o c c} \varepsilon(\mathbf{k})
$$

where the sum is over the occupied states. From the value of $E_{0}$ we can obtain the expectation value of $C_{i}^{+} C_{j}$,

$$
\left\langle C_{i}^{+} C_{j}\right\rangle_{0}=-\frac{E_{0}}{6 \bar{t} N_{0}},
$$

which is independent of the value of $\bar{t}$.

To obtain the expectation value of $\langle V\rangle$, we first notice that since the set $\left\{\delta t_{i, j}\right\}$ are randomly distributed, $\langle\mathbf{k}$ $V|\mathbf{k}\rangle=0$, and it is necessary second order perturbation theory in order to get a correction to the expectation value of $H_{0}$,

$$
E_{2} \simeq-\sum_{\mathbf{k}}^{o c c}\left\langle\mathbf{k}\left|V G_{0}\left(\varepsilon_{\mathbf{k}}\right) V\right| \mathbf{k}\right\rangle
$$

where

$$
G_{0}(\hbar \omega)=\sum_{\mathbf{k}} \frac{|\mathbf{k}\rangle\langle\mathbf{k}|}{\hbar \omega-\varepsilon_{\mathbf{k}}}
$$

with 
is the Green function of the perfect crystal. Because the values $\left\{\delta t_{i, j}\right\}$ are not correlated, it is easy to obtain the expression,

$$
E_{2}=-a_{2} \sum_{\langle i, j\rangle}\left(\bar{t}-t_{i, j}\right)^{2}
$$

with

$$
a_{2}=-\frac{1}{2 N_{0}^{2}} \sum_{\mathbf{k}}^{o c c} \sum_{\mathbf{k}^{\prime}}\left(6+\frac{\varepsilon\left(\mathbf{k}+\mathbf{k}^{\prime}\right)}{\bar{t}}\right) \frac{1}{\varepsilon(\mathbf{k})-\varepsilon\left(\mathbf{k}^{\prime}\right)} .
$$

Adding $E_{0}$ and $E_{2}$, we obtain the following expression for the energy of the system,

$$
E \simeq-2\left\langle C_{i}^{+} C_{j}\right\rangle_{0} \sum_{\langle i, j\rangle} \cos \frac{\theta_{i j}}{2}-a_{2} \sum_{\langle i, j\rangle}\left(\bar{t}-t_{i, j}\right)^{2}
$$

In figure 5 we plot $\left\langle C_{i}^{+} C_{j}\right\rangle_{0}$ and $a_{2}$ as a function of the electron density $x$. The first term in the above expression is a ferromagnetic coupling for the core spins and the second term is an antiferromagnetic coupling. Note that $\left\langle C_{i}^{+} C_{j}\right\rangle_{0}$ is only linear on $x$ at small values of the electron concentration.

\section{MONTE CARLO RESULTS WITH THE APPROXIMATED DOUBLE EXCHANGE ENERGY}

In figure 2 we plot, for $x=0.1, x=0.25$ and $x=0.3$, the absolute value of the magnetization, $M$, as a function of the temperature, $T$, for the case where the internal energy is obtained by using the approximated DE energy, equation (22), for comparing with the case in which it is obtained by diagonalizing the DE Hamiltonian. The difference between both curves is very small, and the corresponding critical temperatures are practically the same, see figure 11. Therefore we conclude that equation (22) is a good approximation for the energy of the DE model.

Using equation (22), for the DE energy, we can perform MC simulations in much bigger systems, and obtain more precise values of the critical temperatures. In figure 6 we plot the magnetization as a function of the temperature for a sample of size $N=20$. For this size the critical temperature is obtained with a big precision. We have checked that for $N=20$ the values of the critical temperatures are accurate in two digits and that it is not worthwhile to increase more the size of the system. We have performed MC simulations for different values of the electron concentration and, in figure 1, we have plotted the magnetic phase diagram.

Kubo and Ohata [7] have developed an expression for the DE energy which coincides with $E_{0}$. In order to know the importance of $E_{2}$ in the calculation of the value of the critical temperature, we have also computed the value of
$M$ as a function of $T$ by using only $E_{0}$ for the internal energy, see figure 6. Comparing these results with the ones obtained with the approximated DE energy, $E_{0}+E_{2}$, we estimate that the inclusion of $E_{2}$ in the internal energy lowers the critical temperature more than a $20 \%$.

In Fig.7, we plot for different values of the concentration $x$, the statistical average of the absolute value of the hopping amplitude, $\left|t_{i, j}\right|$, as a function of the temperature. This quantity is proportional to the electron bandwidth. We find that $\left\langle\left|t_{i, j}\right|\right\rangle$ is a continuous function of $T$, and there are not signals of any change in the electronic structure near $T_{c}$.

The bandwidth near $T_{c}$ is around 1.15 times bigger than the bandwidth in the $T \rightarrow \infty$ limit. Li et al 15 have obtained that in the $T \rightarrow \infty$ limit of the cDE model, only $\sim 0.5 \%$ of the electron states are localized. In order to know the difference between the density of states at $T \sim T_{c}$ and at $T \rightarrow \infty$, we have calculated the statistical average of the electron density of states. We find that the number of electron states with energies between $-W\left(T_{c}\right)$ and $-W(T \rightarrow \infty)$ is less than $1 \%$ of the total number of states. Therefore, even if all these states were localized, the percentage of localized states near $T_{c}$ is not larger than $2 \%$. We conclude that in the DE models it is not possible to relate the metal-insulator transition with the ferro-paramagnetic one.

The critical temperatures we obtain are around 1.5 times smaller than the obtained in mean field theory by Kubo and Ohata [7]. However, it is around 8 times smaller than the obtained by Millis et al. [8] using also a mean field approximation. We think that the difference occurs because they use a lineal dependence, see Fig. 5, of the kinetic electron energy which overestimate the value of $\left\langle C_{i}^{+} C_{j}\right\rangle_{0}$, and therefore the value of $T_{c}$.

From Kubo and Ohata model (4), it is clear that $T_{c}$ scales with $t\left\langle C_{i}^{+} C_{j}\right\rangle_{0}$ and the magnetization versus $T / T_{c}$ curve is independent of the electron concentration $\mathrm{x}$. But there is no reason to think that this must be the case when the second term, $E_{2}$, of our approach is included in the calculations. However, we have found that the $M$ versus $T / T_{c}$ curves almost coincide (see Fig. 6). This fact is not only found in the frame of our approximation to the DE energy but also when the DE and cDE Hamiltonians are diagonalized.

The presented results are in qualitatively agreement with those obtained by Dagotto et al [16]. However our estimations of $T_{c}$ are $\sim 1.5$ times bigger. The discrepancy is due to the difference in the criterion used to obtain $T_{c}$. In reference [16] the $T_{c}$ is defined as the temperature where the spin-spin correlation function in real space becomes zero at the maximum distance available in the unit cell. They used a unit cell of size $6 \times 6 \times 6$. Our criterium is based in the change of sign of the second derivative of $M$ with respect to $T$. We have checked that both criteria give the same $T_{c}$ in big unit cells, but the criterium based on the correlation function underestimate $T_{c}$ in small unit cell calculations. This is clear in Fig.8 where we plot the spin-spin correlation function versus 
distance for several temperatures and for two unit cells with $N=6$ and $N=10$. The electron concentration is $x=0.14$ to compare with the results presented in reference [16]. In the case of $N=6$ it seems that the critical temperature is smaller than $T=1 / 15$, because the correlation function is zero at a separation of $8 a$. However, when $N=10$ it is clear that the ferromagnetic correlation at $T=1 / 15$ is not zero at $8 a$, and in fact it seems that at this temperature the system is still ferromagnetic. On the other hand, our criterium overestimate the $T_{c}$ for small unit cells and the possibility of increasing the size leads to more realistic values of the critical temperature.

To compare with the experimental critical temperatures, it is necessary an estimation of $\bar{t}$. The experimental bandwidth is around $1-4 \mathrm{eV}$ [2], and therefore the value of $\bar{t}$ is around $0.08-0.3 \mathrm{eV}$. With this result our estimate of the critical temperature is between $150 \mathrm{~K}$ and $500 \mathrm{~K}$ at $x \sim 0.2$. This value of $T_{c}$ is in the range of the observed experimentally.

\section{CONCLUSIONS}

Using Monte Carlo techniques, we have obtained the magnetic phase diagram of the double exchange Hamiltonian. Comparing the results obtained from the double exchange Hamiltonian with a complex hopping (cDE) and with its absolute value (DE) we have found that the Berry phase of the hopping has a negligible effect in the magnetic critical temperature. This implies that it is possible to choose wave functions phase factors that counteract the phase of the hopping such that it is not relevant in the $\mathrm{MC}$ calculations. To avoid the limitations on the size of the systems studied we have developed a second order perturbative approach to calculate the electron kinetic energy without diagonalizing the Hamiltonian. Within this approach we have calculated the critical temperature for a bigger size of the system $(N=20)$ and, consequently, more accurately. The values of $T_{c}$ obtained are in the range of the experimental ones and in qualitative agreement with those in ref. [16. Neither the average of the electron density of states nor the kinetic energy reveal any strange behavior near the magnetic critical temperature. We conclude that, in order to understand the metal-insulator transition which occurs in the manganites, another effects, not included in the double exchange Hamiltonian, should be taken into account.

Acknowledgments. This work was supported by the CICyT of Spain under Contract No. PB96-0085, and by the Fundación Ramón Areces. Helpful conversations with F. Guinea, R. Ramírez, S. Das Sarma and J.A. Vergés are gratefully acknowledged.
[1] R.M. Kusters et al., Physica (Amsterdam) 155B, 362 (1989); K. Chahara et al. Appl. Phys. Lett. 63, 1990 (1993); R. von Helmolt et al. Phys. Rev. Lett. 71, 2331 (1993); S. Jin et al. Science 264, 413 (1994).

[2] See for example, A.P. Ramirez, J. Phys.: Condens. Matter 9, 8171-8199 (1997); J.M.D. Coey, M. Viret and S. von Molnar, Adv. in Phys., in press.

[3] C. Zener, Phys. Rev. 82, 403 (1951).

[4] P.W. Anderson and H. Hasegawa, Phys. Rev 100, 675 (1955).

[5] P.G. deGennes, Phys. Rev. 118, 141 (1960).

[6] E. Müller-Hartmann and E. Dagotto, Phys. Rev. B 54, R6819 (1996).

[7] K. Kubo and N. Ohata, J. Phys. Soc. Jpn. 33, 21 (1972).

[8] A.J. Millis, P.B. Littlewood and B.I. Shraiman, Phys. Rev. Lett. 74, 5144 (1995).

[9] H. Röder, J. Zang and A.R. Bishop, Phys. Rev. Lett. 76, 1536 (1996); J. Zang, A.R. Bishop and H. Röder, Phys. Rev. B 53, R8840 (1996).

[10] A.J. Millis, B.I. Shraiman and R. Mueller, Phys. Rev. Lett. 77175 (1996); A.J. Millis, R. Mueller and B.I. Shraiman, Phys. Rev. B 545405 (1996).

[11] S. Yunoki, J. Hu, A.L. Malvezzi, A. Moreo, N. Furukawa and E. Dagotto, Phys. Rev. Lett. 80, 845 (1998).

[12] D. Arovas and F. Guinea, cond-mat/9711145.

[13] Monte Carlo methods in Statistical Physics, edited by K. Binder (Springer-Verlag, Berlin, 1979).

[14] P.A. Serena, N. García and A. Levanyuk, Phys. Rev. B, 47, 5027 (1993).

[15] Q. Li, J. Zang, A.R. Bishop and C.M. Soukoulis, Phys. Rev. B, 56, 4541, (1997).

[16] E. Dagotto, S. Yunoki, A.L. Malvezzi, A. Moreo, J. $\mathrm{Hu}$, S. Capponi, D. Poilblanc and N. Furukawa, condmatt/9709029.

FIG. 1. Critical temperature of the ferro-paramagnetic transition versus concentration of conduction electrons for different approaches. Squares correspond to the Hamiltonian (11) with the hopping given by (2); circles to hopping (3). The curves are related to the calculations made with an approximated expression obtained in order to avoid diagonalization in the MC simulations (ADEE: approximation to the double exchange energy). This fact allows us to increase the size of the system. The line is only a guide to the eye. The critical temperatures obtained are in the range of the experimental ones.

FIG. 2. Absolute value of the magnetization versus the temperature for concentrations $x=0.1,0.25$ and 0.3 . The size of the unit cell is $N=4$. In order to compare we have plotted also the curves obtained with the cDE and DE Hamiltonians as well as with the perturbative approach developed here (ADEE).

FIG. 3. Double exchange energy versus temperature. The lack of difference in these curves implies the result in Fig. A. The corresponding critical temperatures are pointed with an arrow. 
FIG. 4. Bandwidth (a) and chemical potential (b) versus temperature. In the disordered case, $T \rightarrow \infty, W$ should be equal to 8 . Here, in the paramagnetic phase, $W>8$ because the transition is second order (see text for details). Note that the curves are continuous near $T_{c}$ which is pointed with an arrow in each case.

FIG. 5. Coefficients used in the perturbative approach to the DE Hamiltonian versus concentration (see eq. (22)).

FIG. 6. Magnetization as a function of $T / T_{c}$ as obtained using the $0^{t h}$ order approximation (dashed line), $E_{0}$ in eq. (22), and the $2^{\text {nd }}$ order approximation (continuous line) to the double exchange energy (ADEE), eq. (22). The critical temperature taken for reference is the one that corresponds to the ADEE model. In the case of the $0^{\text {th }}$ order approximation, the $M$ versus $T / T_{c}$ curve is independent of the electron concentration. In the ADEE model, there is no reason for this independence; however, we have found that $M\left(T / T_{c}\right)$ is practically independent of $\mathrm{x}$. To show this, the $M\left(T / T_{c}\right)$ curve has been plotted for various concentrations $(x=0.1,0.2,0.3$, 0.4 and 0.5$)$.

FIG. 7. Average of the absolute value of the hopping amplitude, $\left\langle\left|t_{i, j}\right|\right\rangle$, versus temperature for $N=20$ and concentrations $x=0.1,0.25$ and 0.3 . This average is proportional to the bandwidth. We recover the continuous behavior that we found on Fig.4.

FIG. 8. The spin-spin correlation versus distance is plotted for different temperatures. In (a) $N=6$ and in (b) $N=10$. For these calculations we have used the perturbative approach. If the criterium to obtain $T_{c}$ is that the spin-spin correlation becomes zero, a smaller size of the system leads to an underestimation of the critical temperature. 


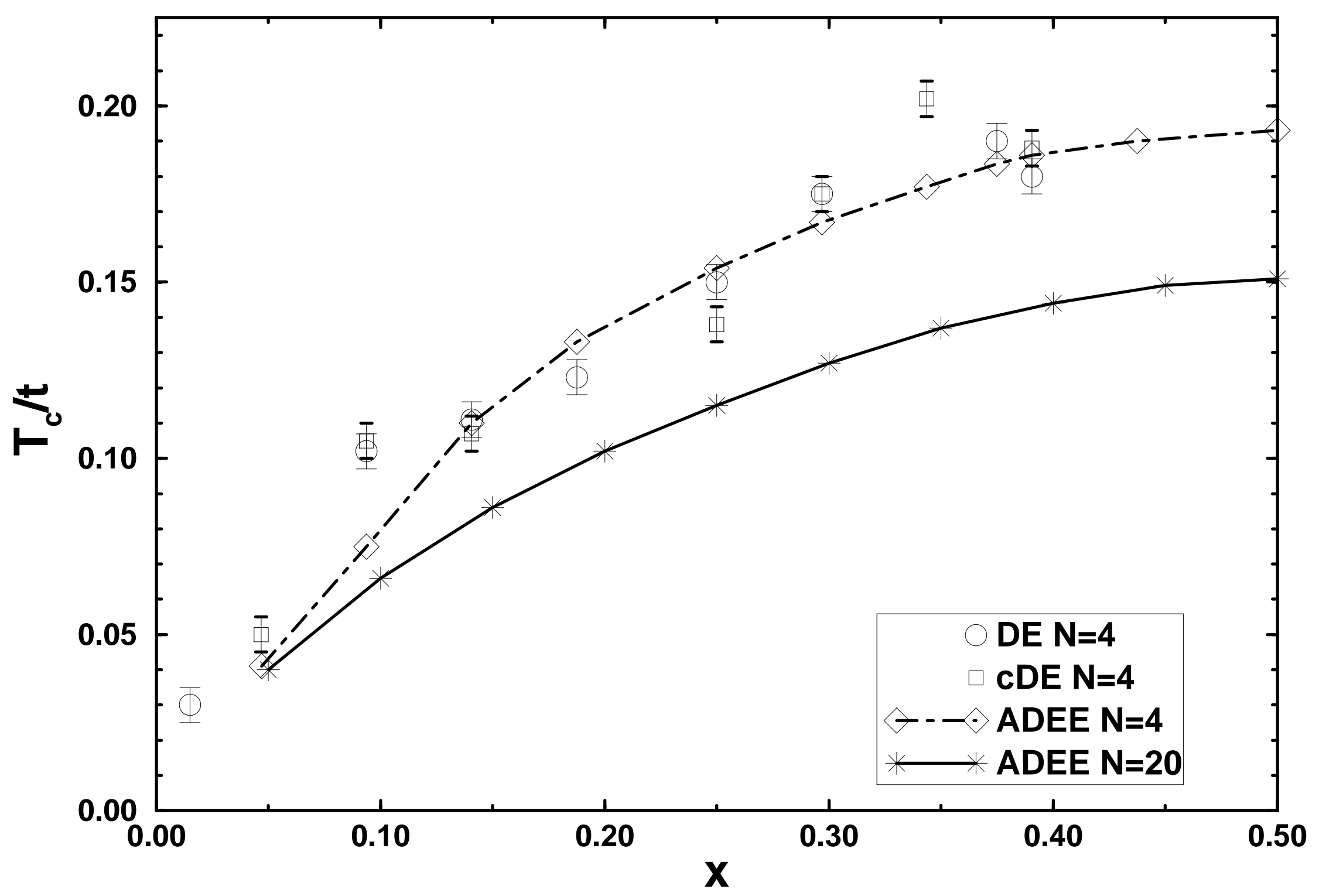

Fig.1 M.J. Calderon and L. Brey 


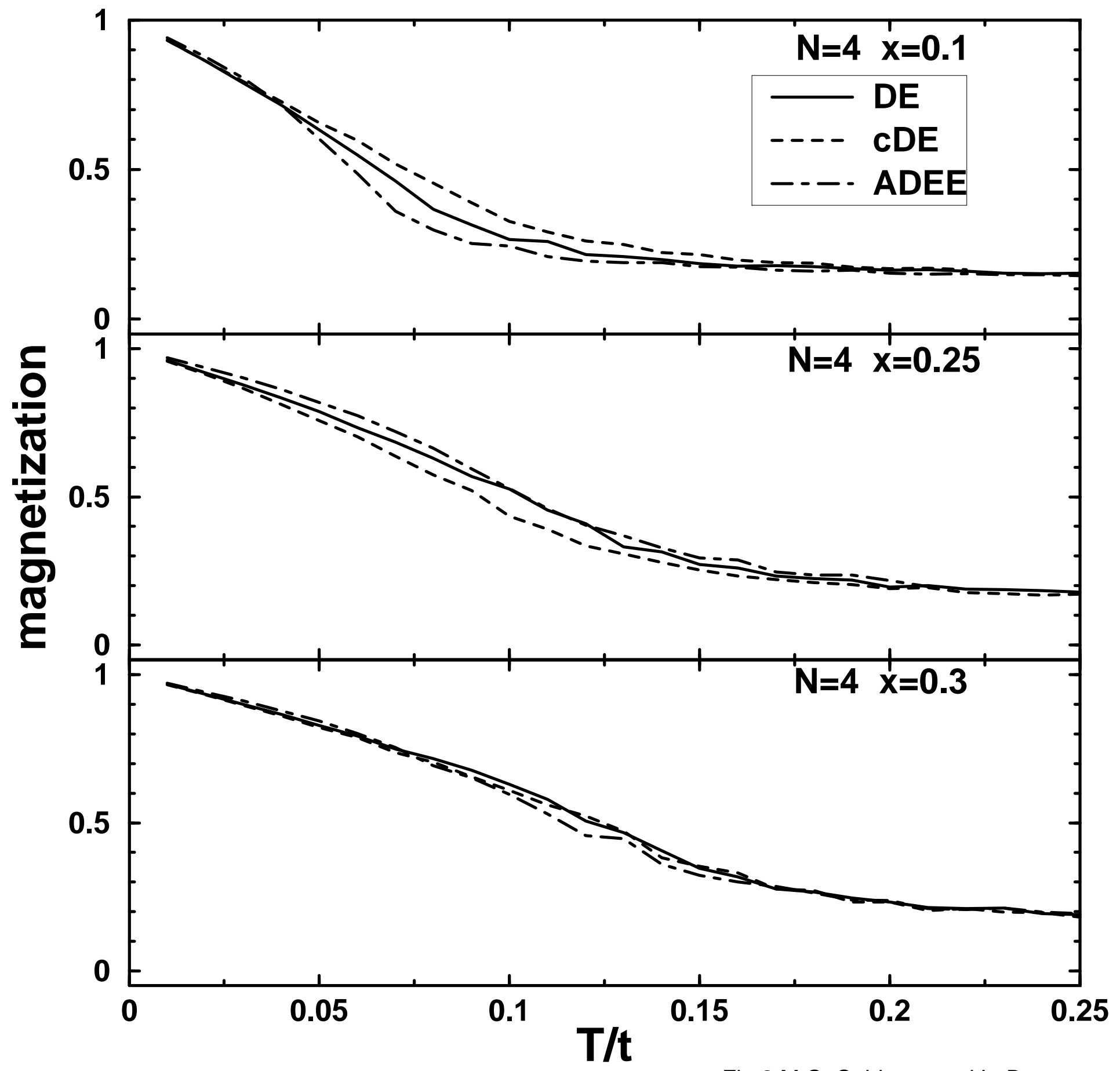

Fig.2 M.C. Calderon and L. Brey 


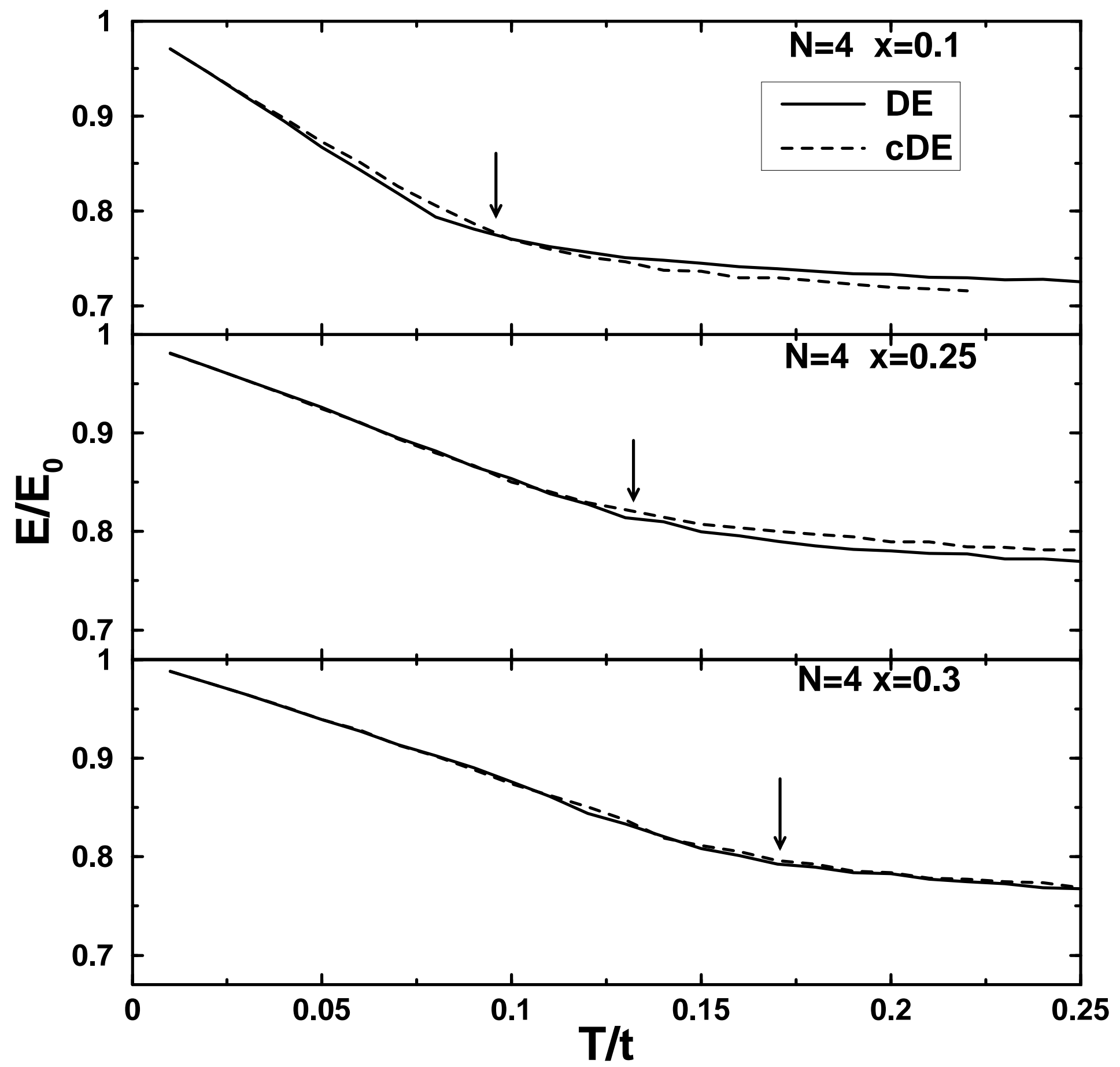

Fig. 3 M.J. Calderon and L. Brey 


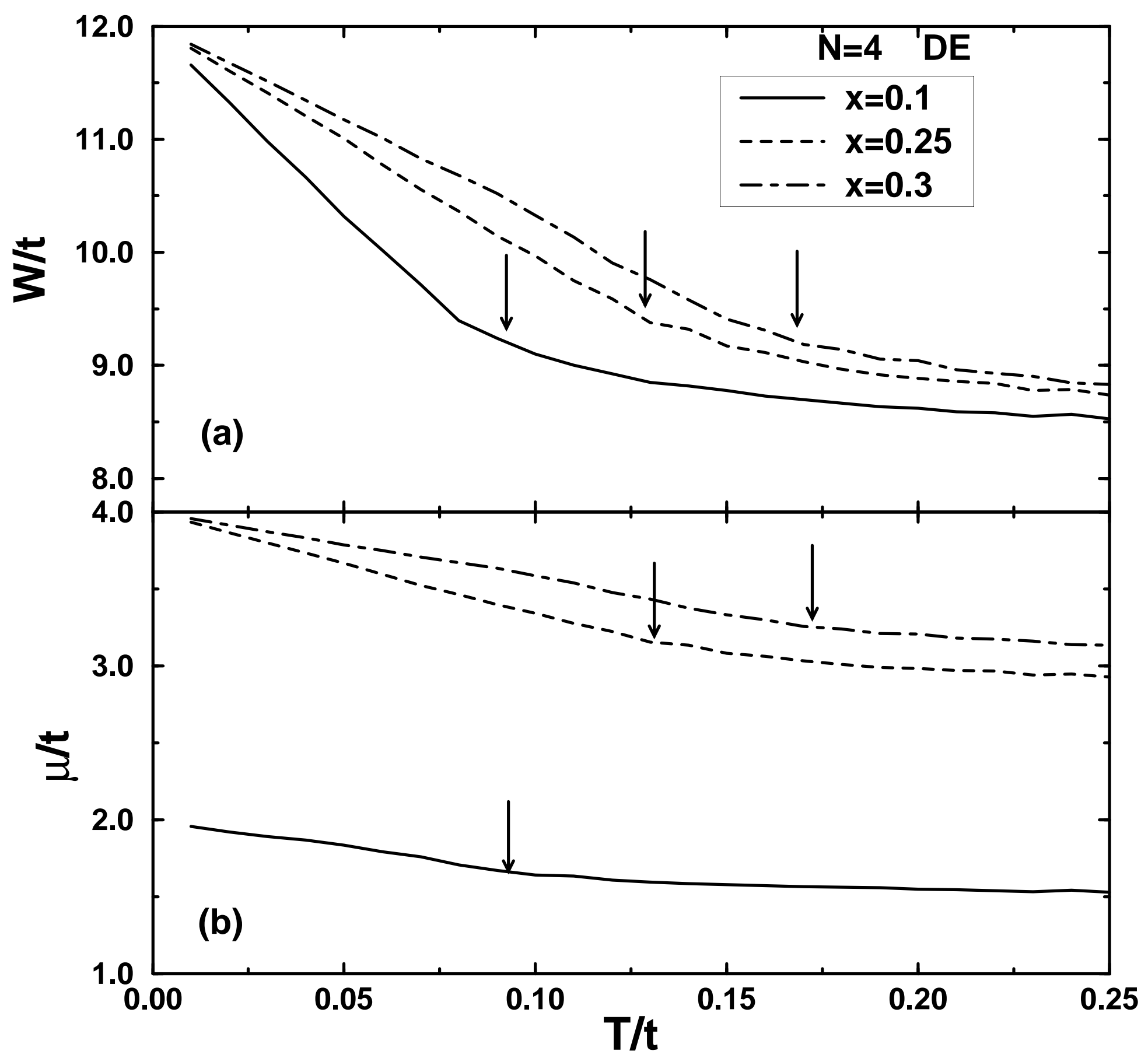

Fig.4 M.J. Calderon and L. Brey 


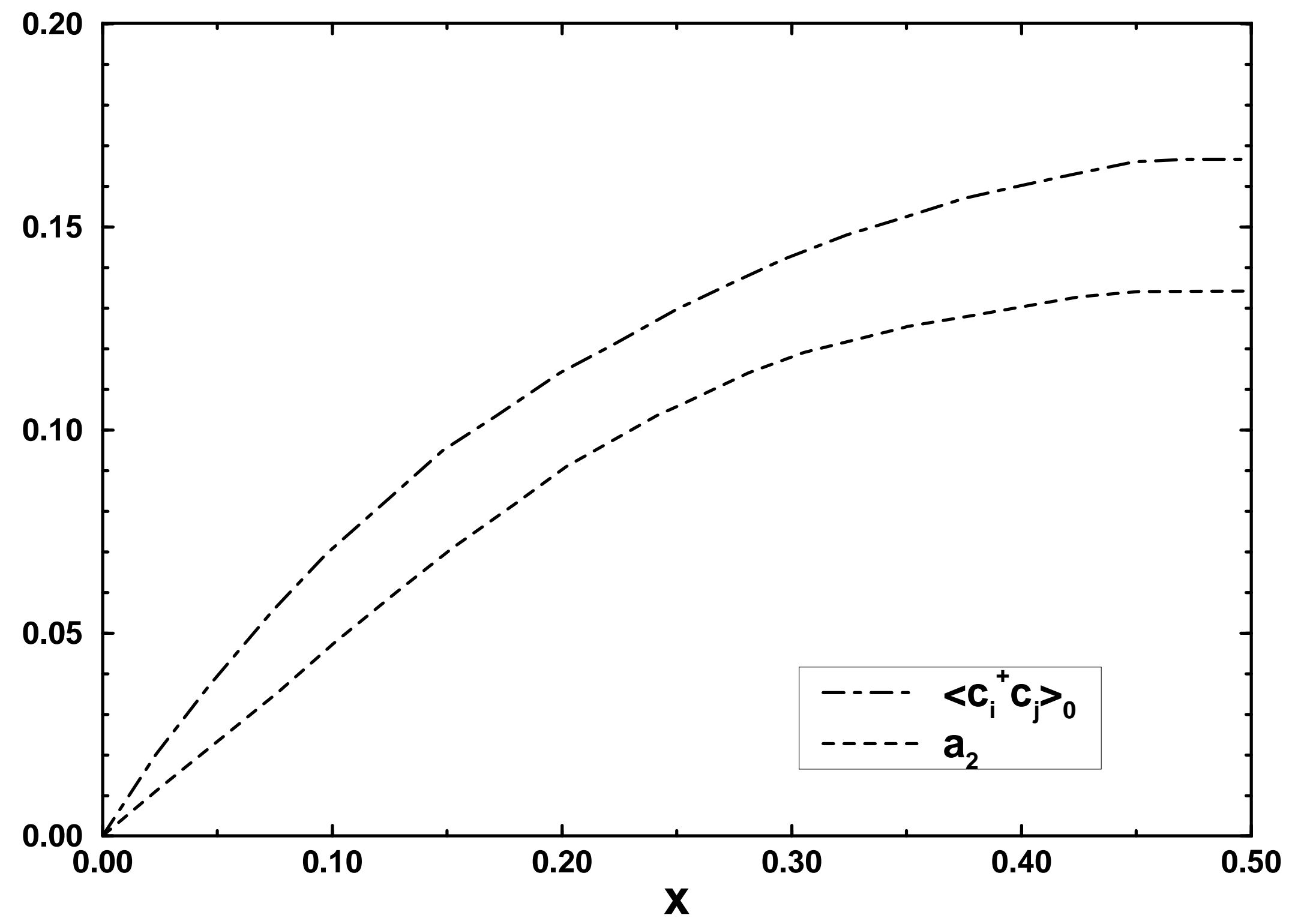

Fig.5 M.J. Calderon and L. Brey 


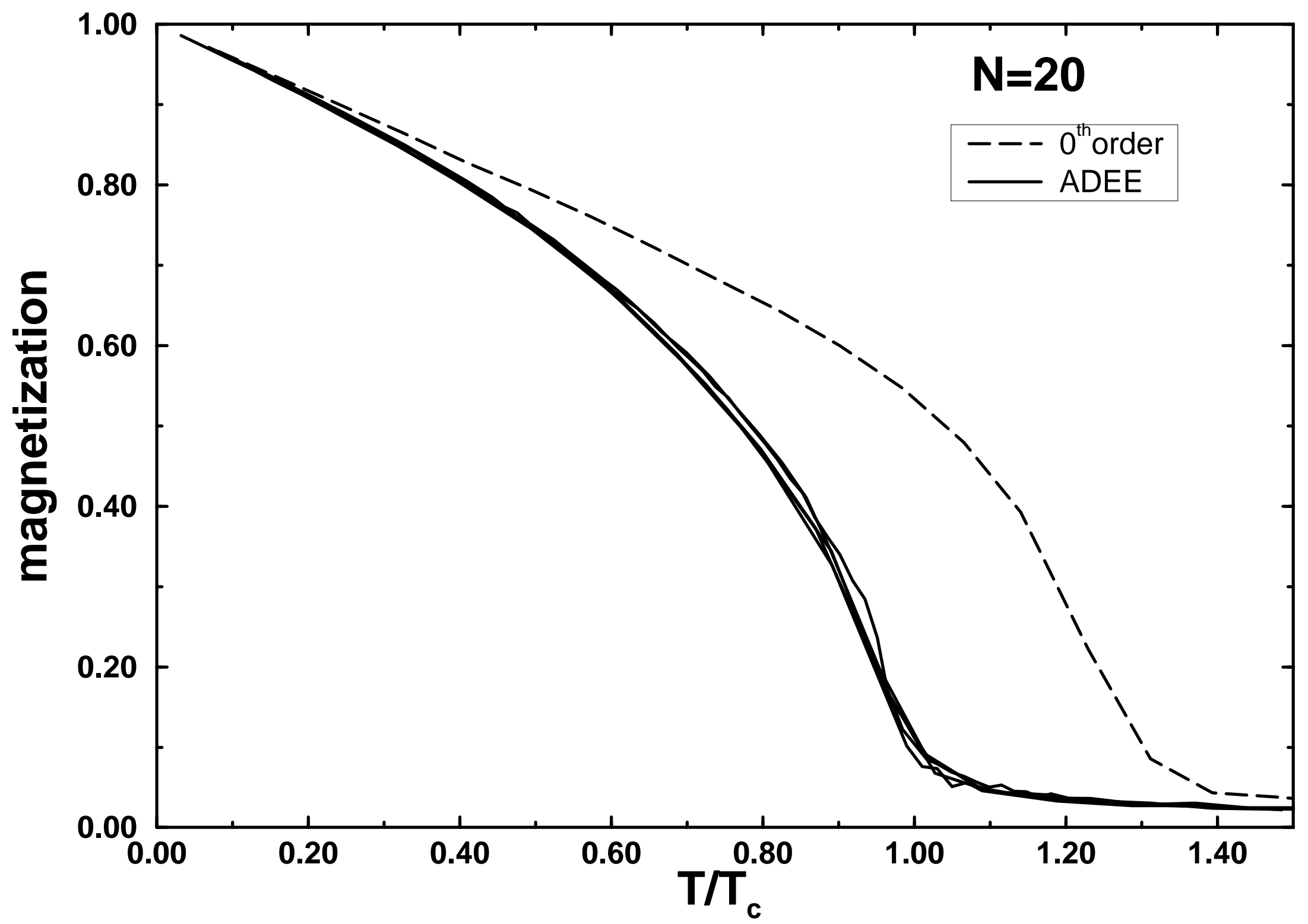

Fig.6 M.C. Calderon and L. Brey 


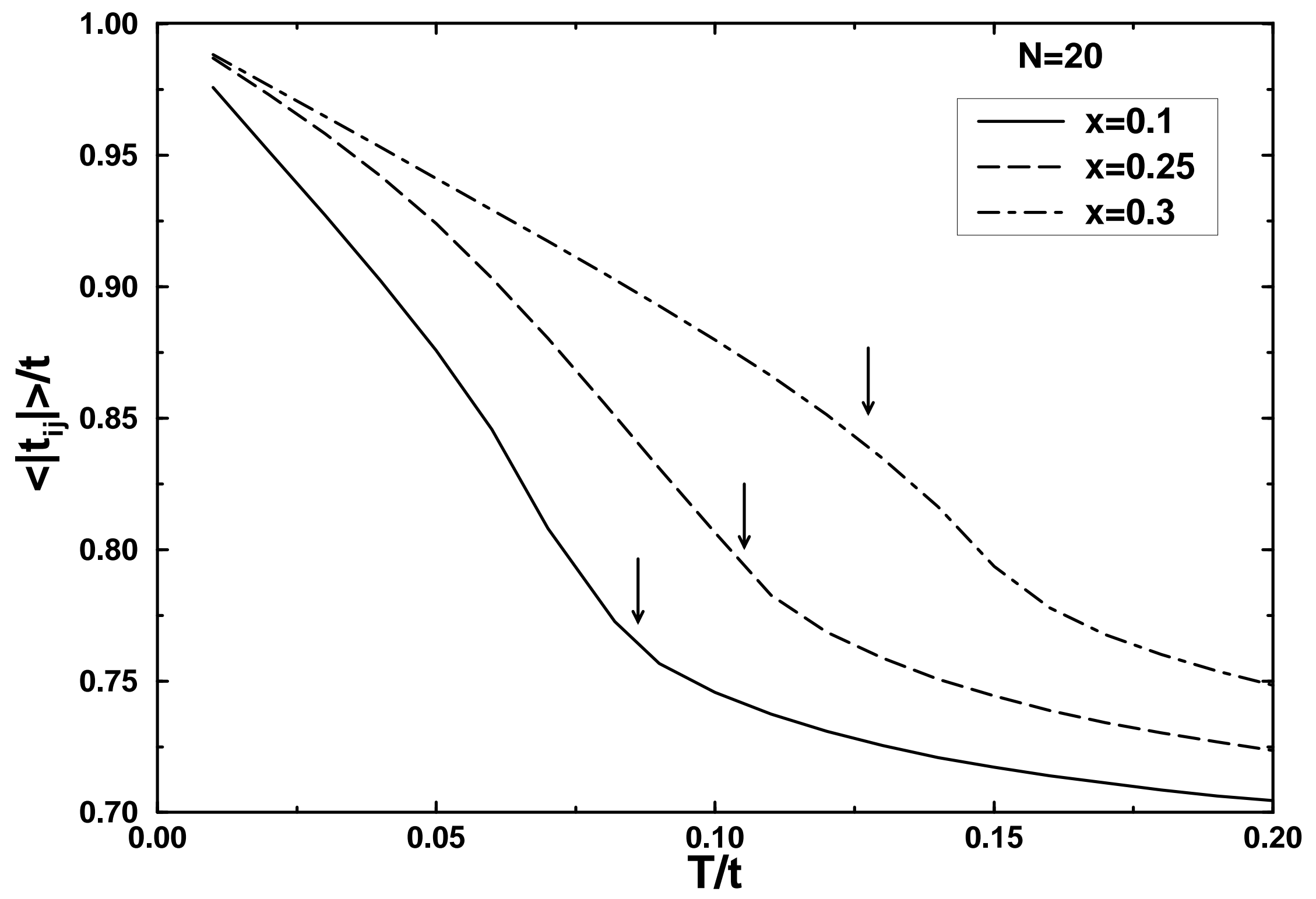

Fig.7 M.J. Calderon and L. Brey 


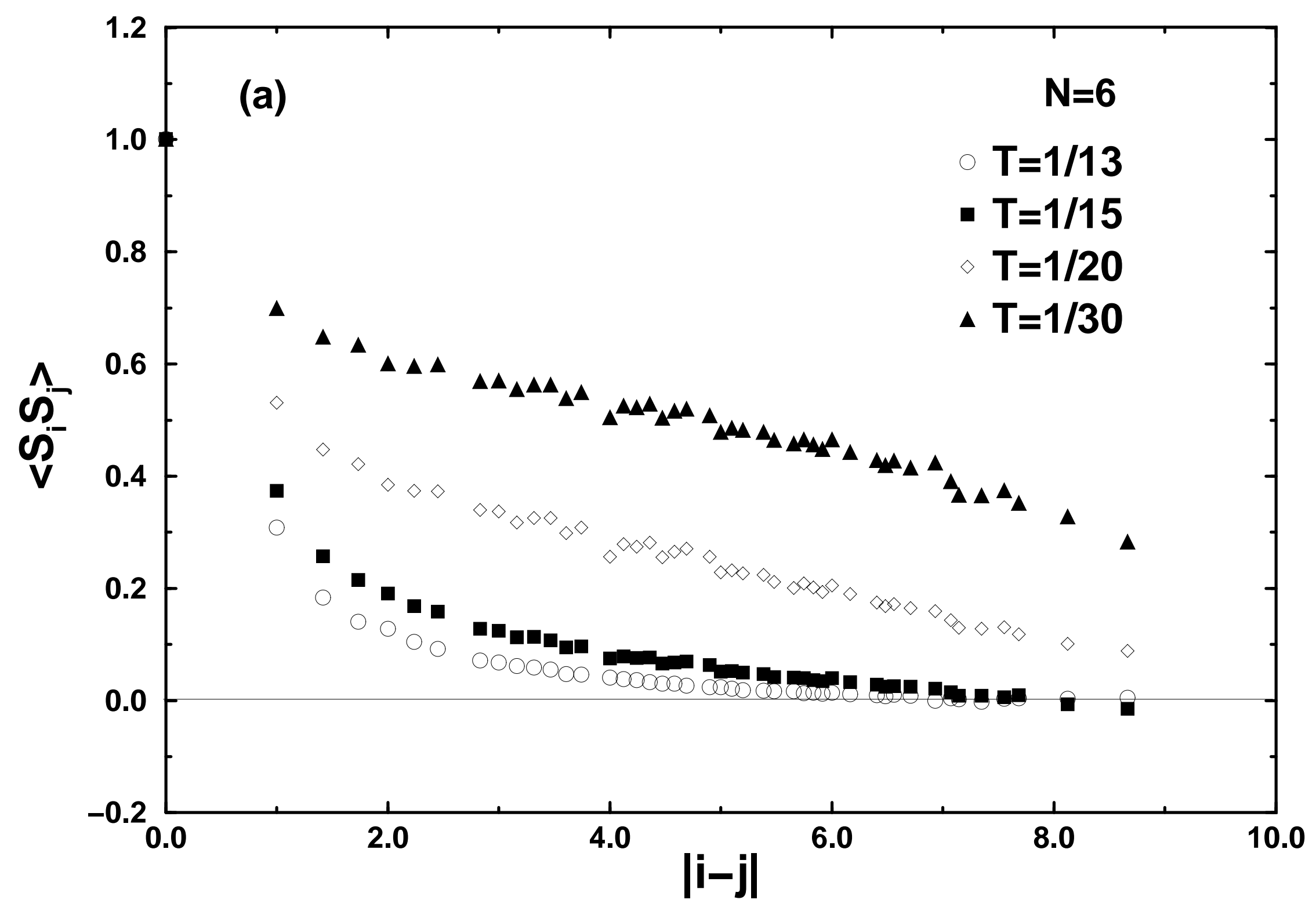

Fig.8a M.J. Calderon and L. Brey 


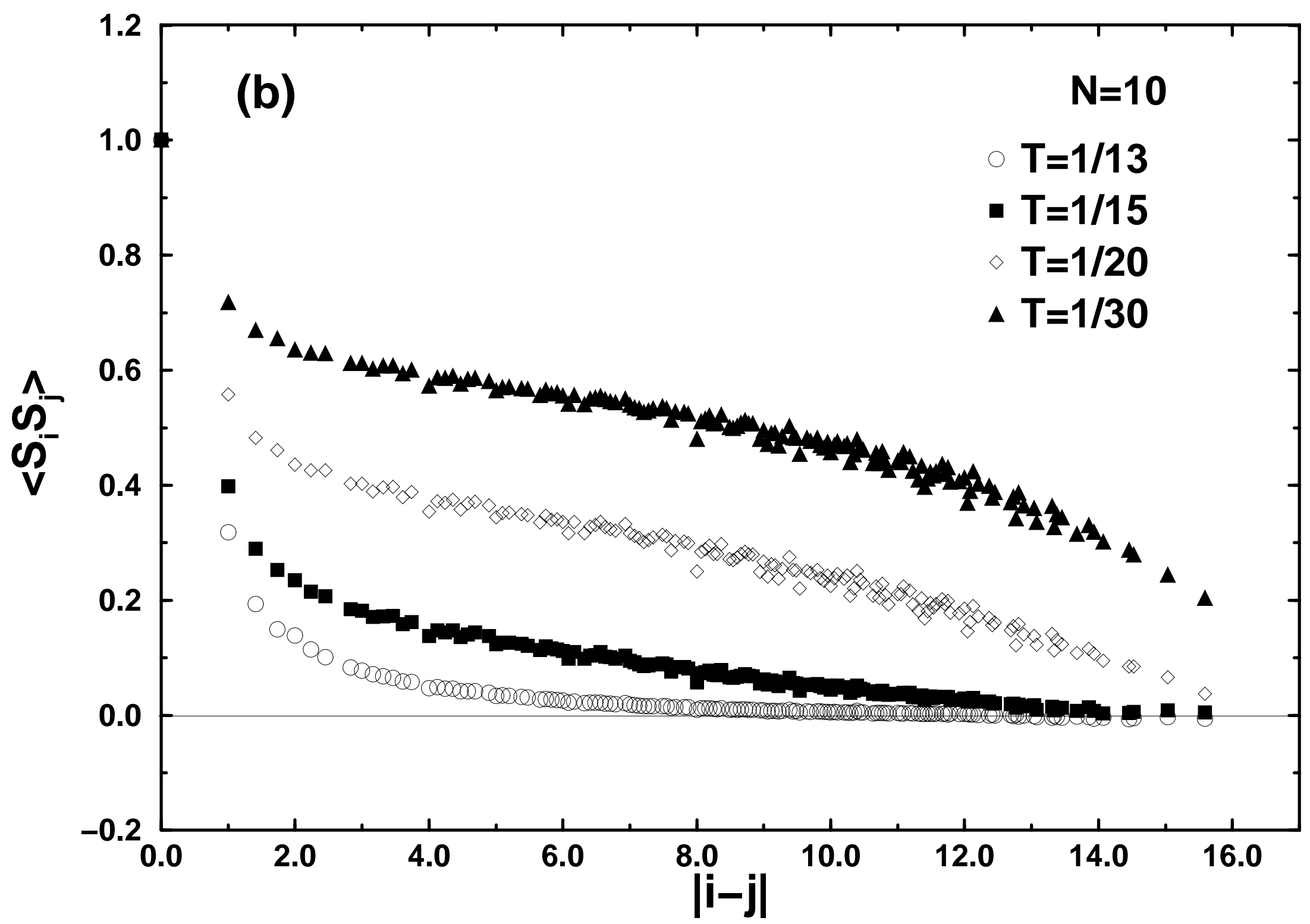

Fig.8b M.J. Calderon and L. Brey 